\title{
Seletividade do HeRBicida SAFlufenacil A Eucalyptus urograndis ${ }^{1}$
}

\author{
Selectivity of Saflufenacil to Eucalyptus urograndis
}

PEREIRA, M.R.R. ${ }^{2}$, MARTINS, D. ${ }^{3}$, RODRIGUES, A.C.P. ${ }^{4}$, SOUZA, G.S.F. ${ }^{5}$ e CARDOSO, L.A. ${ }^{6}$

\begin{abstract}
RESUMO - As áreas com plantios florestais estão constantemente em expansão, e a interferência das plantas daninhas alteram o crescimento e desenvolvimento de plantas de eucalipto, pois estas competem por água, nutrientes, espaço e luz. Objetivou-se com este estudo avaliar a seletividade do herbicida saflufenacil, aplicado com e sem óleo mineral Dash, em plantas de Eucalyptus urograndis, em diferentes locais de aplicação (planta, solo e solo + planta). O delineamento experimental adotado foi o inteiramente casualizado, com quatro repetições, constituído de um fatorial 3 x 11 , sendo três locais de aplicação do herbicida na planta e 11 doses do herbicida saflufenacil combinadas ou não com o adjuvante Dash. Cada parcela constou de um vaso com uma planta. Os tratamentos usados foram: 0, 25, 25+Dash (0,5\% v/v), 50, 50+Dash (0,5\% v/v), 75, 100, 125, 150, 175 e 200 g i.a. ha-1. Foram realizadas avaliações visuais de fitointoxicação durante 56 dias após aplicação (DAA). Avaliouse, ainda, a altura, o diâmetro de caule e a biomassa seca das plantas. Todas as doses testadas de saflufenacil sem adjuvante mostraram-se seletivas às plantas de E. urograndis, independentemente do local de aplicação. A adição de Dash aos tratamentos proporcionou injúrias severas às plantas nas avaliações iniciais, quando aplicado sobre as plantas, com a recuperação total destas no fim do ensaio, aos 56 DAA. A aplicação do herbicida em mistura com adjuvante somente no solo não causou intoxicação às plantas de eucalipto.
\end{abstract}

Palavras-chave: controle químico, eucalipto, fitotoxicidade, local de aplicação.

\begin{abstract}
Forest plantation areas are constantly expanding. However, weed interference affects the growth and development of eucalyptus plants, as they compete for water, nutrients, space, and light. The objective of this study was to evaluate the selectivity of the herbicide saflufenacil, applied with or without the mineral oil Dash, on Eucalyptus urograndis at different application sites (plant, soil and soil + plant). The experimental design was completely randomized, with four replications, consisting of a $3 \times 11$ factorial, with three sites for herbicide application on the plant and eleven saflu fenacil doses combined or not with the adjuvant Dash. Each plot consisted of a vase with a plant. Treatments were 0, 25, $25+\operatorname{Dash}(0.5 \% \mathrm{v} / \mathrm{v}), 50,50+\operatorname{Dash}(0.5 \% \mathrm{v} / \mathrm{v}), 75,100,125$, 150,175 and $200 \mathrm{~g}$ a.i. ha ${ }^{-1}$. Visual evaluations of plant intoxication were carried out for 56 days after application (DAA). Height, stem diameter, and dry biomass of the plants were also evaluated. The saflufenacil doses tested without adjuvant proved to be selective for the plants of $\boldsymbol{E}$. urograndis, independent of the application site. The addition of Dash to the treatments caused severe injuries to the plants at the initial assessments, when applied on the plants, with full recovery at the end of the test at 56 DAA. Herbicide application combined with the adjuvant only in the soil did not cause plant toxicity to the eucalyptus plants.
\end{abstract}

Keywords: chemical control, eucalyptus, plant toxicity, application site.

1 Recebido para publicação em 17.2.2011 e aprovado em 22.3.2011.

2 Engenheira Florestal, Pós-Doutoranda em Agronomia, Faculdade de Ciências Agronômicas, Universidade Estadual Paulista “Júlio de Mesquita Filho" - FCA/UNESP, Caixa Postal 237, 18603-970 Botucatu-SP, <mariarenata10@hotmail.com>; ${ }^{3}$ Professor Adjunto, Dep. de Produção Vegetal, FCA/UNESP, Botucatu-SP, ${ }^{4}$ Pós-Doutoranda, Universidade Estadual do Oeste do Paraná - UNIOESTE, Caixa Postal 1008, 85960-000 Marechal Cândido Rondon-PR; ${ }^{5}$ Doutorando em Agronomia/Agricultura, FCA-UNESP, BotucatuSP; ${ }^{6}$ Engo-Agro ${ }^{-}$, Dr., Pesquisador FAO, Bissau/Guiné-Bissau.

Planta Daninha, Viçosa-MG, v. 29, n. 3, p. 617-624, 2011 


\section{INTRODUÇÃO}

As espécies florestais como o eucalipto e o pínus são plantas de rápido crescimento, possuem tolerância ao frio e apresentam poucas exigências em fertilidade de solo. Apesar dessas vantagens, que fazem com que elas sejam escolhidas para plantio em relação a várias espécies florestais disponíveis, certos cuidados devem ser tomados para que haja bom desenvolvimento das plantas e, por fim, alta produtividade florestal.

De todas as práticas de manejo adotadas visando à alta produtividade da floresta, o controle de plantas daninhas é uma das mais efetuadas, principalmente nos primeiros anos da plantação. As plantas daninhas, que muitas vezes são nativas da região, outras vezes gramíneas de pastagens, são bem adaptadas às condições adversas de nosso meio ambiente, podendo sobressair às pequenas mudas florestais recém-plantadas e que ainda estão em processo de estabelecimento. As plantas daninhas também podem ser hospedeiras de pragas e doenças, porém o principal dano que elas causam nas plantações jovens das florestas é a competição (Garcia et al., 2007).

As plantas daninhas são consideradas o maior problema mundial nos reflorestamentos, pois estas competem por água, luz, nutrientes e podem, ainda, apresentar efeitos alelopáticos (Toledo et al., 2003). Em áreas de recuperação no cerrado, locais antes ocupados por pastagem ou mata nativa, essas plantas surgem com grande agressividade. A interferência imposta pelas plantas daninhas às culturas florestais é mais severa sobretudo na fase inicial de crescimento, ou seja, até cerca de um ano após o transplante das mudas (Constantin et al., 2000).

Mesmo que as plantações florestais sejam provenientes de áreas, climas, solos e composição vegetal diferentes, estudos apontaram que a maior influência da competição dá-se nos primeiros anos, devendo-se monitorar o desenvolvimento da comunidade infestante, o que poderá resultar em melhor tratamento no que se refere a custo-beneficio, que pode ser físico ou químico, bem como o manejo delas somente na linha depois da fase de estabelecimento da cultura e por um período necessário (Cantarelli, 2006).
Assim, o uso de herbicidas em reflorestamento torna-se uma prática indispensável e amplamente utilizada devido ao seu alto rendimento e eficiência, em comparação com as demais. Os produtos utilizados devem ter amplo espectro de ação para as plantas daninhas, atingir o maior número de espécies possivel e não afetar a cultura em questão, sendo seletivo a estas (Victoria Filho, 1987).

É necessária a avaliação dos efeitos de um herbicida nas diferentes culturas para uma melhor utilização destes, como o saflufenacil (fórmula molecular: $\mathrm{C}_{17} \mathrm{H}_{17} \mathrm{ClF}_{4} \mathrm{~N}_{4} \mathrm{O}_{5} \mathrm{~S}$ ), um latifolicida eficiente que está em fase de registro para diversas culturas no Brasil, incluindo a cultura de eucalipto. Ele tem ação de contato, que vem complementar o espectro de plantas daninhas controladas pelo glyphosate. Provoca inicialmente sintomas como manchas verdeescuras nas folhas, que progridem para necrose e secamento das folhas (BASF, 2010).

De acordo com Soltani et al. (2010), as espécies suscetiveis são controladas por esse herbicida através da inibição da enzima protoporfirinogênio oxidase (PROTOX), que é absorvida por raízes e folhagem das plantas. É principalmente translocado no xilema e tem limitada mobilidade no floema. A seletividade ocorre basicamente pela metabolização da molécula do herbicida (Grossmann et al., 2010).

Resultados encontrados por Geier et al. (2009) mostraram que o herbicida saflufenacil, quando aplicado de forma isolada e em pós-emergência, controlou eficientemente Amaranthus hibridus, Amaranthus viridis e Descurainia sophia, que são plantas daninhas também encontradas em áreas de reflorestamento. De acordo com Davis et al. (2010), na dessecação em área total o herbicida saflufenacil foi o único tratamento que reduziu em mais de $90 \%$ a densidade de buva (Conyza canadensis), em relação ao 2,4-D + glyphosate.

A seletividade dos herbicidas, como descrito por Victoria Filho (1987), às plantas pode ocorrer porque o herbicida pode ficar localizado fora do sistema radicular (seletividade toponômica) e da parte aérea da planta (aplicação protegida) ou então devido à sua metabolização pela planta cultivada (seletividade fisiológica). A seletividade depende das características da molécula do herbicida, bem 
como também da tolerância das plantas cultivadas à sua ação fitotóxica (Camargo, 1977). Essa tolerância das plantas à ação dos herbicidas depende de suas caracteristicas morfológicas, estruturais e fisiológicas.

Em estudo realizado por Soltani et al. (2009), o herbicida saflufenacil pode ser utilizado em pré-emergência das plantas, ou seja, com a aplicação no solo, com segurança, em milho em doses de até $200 \mathrm{~g}$ i.a. ha ${ }^{-1}$. Também pode ser utilizado em condição de pós-emergência da cultura do milho, sem a adição de adjuvante (50 ou $100 \mathrm{~g}$ i.a. ha ${ }^{-1}$ ). Em contrapartida, a aplicação desse herbicida em pós-emergência com um adjuvante resultou em prejuízos e perdas de produção de milho no campo.

O herbicida saflufenacil aplicado em préemergência também pode ser usado com segurança em cultivares especificos de ervilha e soja, na dose de $100 \mathrm{~g}$ i.a. ha ${ }^{-1}$. No entanto, não há uma margem de segurança aceitável para as culturas do feijão azuki e branco e em frutas como o cranberry e laranja-lima (Soltani et al., 2010).

Este estudo teve como objetivo avaliar a seletividade do herbicida saflufenacil aplicado com e sem óleo mineral Dash, em plantas de Eucalyptus urograndis em diferentes locais de aplicação (planta, solo e solo + planta).

\section{MATERIAL E MÉTODOS}

O estudo foi instalado na região centrooeste do Estado de São Paulo, entre os meses de julho e outubro, e as mudas foram mantidas em pleno sol (os dados meteorológicos são apresentados na Figura 1).

As mudas de E. urograndis com 100 dias foram transplantadas para vasos plásticos, com capacidade para 3,5 L. O solo utilizado foi classificado como de textura argilosa $(50,4 \%$ de argila, $25,7 \%$ de silte e $23,9 \%$ de areia) e adubado de acordo com as necessidades da cultura do eucalipto, com base na análise química (Tabela 1). Foi conduzida uma planta por vaso, e o delineamento experimental utilizado foi o inteiramente casualizado, com quatro repetições, constituído de um fatorial $3 \times 11$, sendo três locais de aplicação do herbicida na planta e 11 doses combinadas ou não com o adjuvante Dash do herbicida saflufenacil.

A aplicação dos tratamentos foi realizada 15 dias após o transplantio das mudas, quando estas se encontravam em média com $35 \mathrm{~cm}$ de altura e com 7 a 8 pares de folhas, ocorrendo nas seguintes formas: na planta, no solo e no solo + planta. Para aplicação nas plantas, o solo foi coberto com sacos plásticos, para evitar que fosse atingido pela solução. Já para a aplicação no solo adotou-se o mesmo procedimento, evitando que as plantas fossem atingidas pela solução. Os tratamentos usados foram: 0,25 , 25+Dash $(0,5 \% \mathrm{v} / \mathrm{v}), 50,50+\operatorname{Dash}(0,5 \% \mathrm{v} / \mathrm{v})$, 75, 100, 125, 150, 175 e 200 g i.a. ha ${ }^{-1}$.

Foi utilizado um pulverizador estacionário munido de quatro pontas XR 11002 VS, com pressão de $200 \mathrm{kPa}$ e consumo de calda de $200 \mathrm{~L} \mathrm{ha}^{-1}$, para aplicação do herbicida. Nos tratamentos com aplicação de herbicida sobre o solo, foi realizada uma chuva de $5 \mathrm{~mm}$ com um simulador estacionário, após a pulverização.

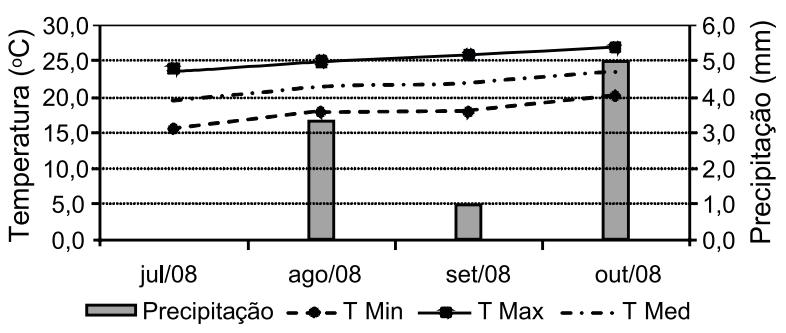

Figura 1 - Médias mensais de temperaturas máxima, média e mínima e precipitação pluviométrica. Botucatu-SP, 2008.

Tabela 1 - Análise química de fertilidade do solo utilizado no estudo. Botucatu-SP. 2008

\begin{tabular}{|c|c|c|c|c|c|c|c|c|c|}
\hline $\mathrm{pH}$ & MO & Presina & $\mathrm{H}+\mathrm{Al}$ & K & $\mathrm{Ca}$ & $\mathrm{Mg}$ & SB & CTC & $\mathrm{V}(\%)$ \\
\hline$\left(\mathrm{CaCl}_{2}\right)$ & $\left(\mathrm{g} \mathrm{dm}^{-3}\right)$ & $\left(\mathrm{mg} \mathrm{dm}^{-3}\right)$ & \multicolumn{6}{|c|}{$\left(\mathrm{mmol}_{\mathrm{c}} \mathrm{dm}^{-3}\right)$} & $v(70)$ \\
\hline 4,8 & 20 & 37 & 40 & 3,1 & 19 & 5 & 27 & 67 & 40 \\
\hline
\end{tabular}


Avaliações visuais de controle foram realizadas aos 1, 7, 14, 21, 42 e 56 dias após a aplicação (DAA) dos tratamentos químicos, por meio de uma escala de notas, na qual 0 (zero) correspondeu a nenhuma injúria e 100 à morte das plantas (SBCPD, 1995). Foram realizadas, no final do estudo, medições de altura, diâmetro do colo e matéria seca das plantas.

Os resultados foram submetidos à análise de variância pelo teste $\mathrm{F}$, sendo as médias dos tratamentos comparadas pelo teste de Tukey a $5 \%$ de probabilidade.

\section{RESULTADOS E DISCUSSÃO}

Na Tabela 2 estão apresentados os resultados de fitotoxicidade verificados aos 1 e 7 DAA do herbicida saflufenacil aplicado de forma isolada, em várias dosagens, e de forma combinada com Dash em três diferentes locais: solo, planta e solo+planta. Com apenas 1 DAA observaram-se elevados níveis de intoxicação, atingindo até $38,25 \%$, nos tratamentos com aplicação de saflufenacil combinado com Dash, quando aplicados sobre a planta e solo + planta, não sendo verificado nenhum sintoma nos outros tratamentos. Sintomas precoces de fitointoxicação em $E$. urophylla também foram observados por Tuffi Santos et al. (2006a) em plantas expostas à aplicação dos herbicidas triclopyr e carfentrazone-ethyl, aos 2 e 3 DAA.

Aos 7 DAA, verificaram-se leves sintomas de fitotoxicidade (variando de 0 a $16,8 \%$ ) com aplicação isolada de saflufenacil, mesmo quando se utilizou a dose de 200 g i.a. ha- ${ }^{-1}$, independentemente do local de aplicação. Nos tratamentos adicionados com Dash, observaram-se valores próximos a $60 \%$ de fitotoxicidade quando a aplicação foi feita sobre a planta ou solo + planta, com a morte dos ponteiros e dos primeiros pares de folhas totalmente expandidas, como também cloroses, que evoluíram para necroses de coloração parda no restante das folhas, não sendo observada fitotoxicidade nas aplicações do herbicida realizadas apenas sobre o solo. As cloroses observadas podem ser reflexo da degeneração dos cloroplastos e/ou inibição da formação da clorofila - efeito semelhante ao relatado para outras espécies de plantas de eucalipto expostas ao glyphosate, conforme mencionado por Tuffi Santos et al. (2008). Pode-se, assim, inferir que, mesmo em altas dosagens, a molécula isolada do herbicida não provocou

Tabela 2 - Porcentagem de intoxicação de Eucalyptus urograndis submetido à aplicação de diferentes doses do herbicida saflufenacil, em três locais de aplicação na planta. Botucatu-SP, 2008

\begin{tabular}{|c|c|c|c|c|c|c|c|}
\hline \multirow{3}{*}{ Tratamento } & \multirow{2}{*}{ Dose } & \multicolumn{3}{|c|}{$1 \mathrm{DAA}$} & \multicolumn{3}{|c|}{$7 \mathrm{DAA}$} \\
\hline & & \multicolumn{3}{|c|}{ Local de aplicação } & \multicolumn{3}{|c|}{ Local de aplicação } \\
\hline & $\left(\mathrm{g}\right.$ i.a. $\left.\mathrm{ha}^{-1}\right)$ & Solo & Planta & Solo + planta & Solo & Planta & Solo + planta \\
\hline Testemunha & - & $0,00(0,00) \mathrm{aA}$ & $0,00(0,00) \mathrm{aB}$ & $0,00(0,00) \mathrm{aC}$ & $0,00(0,00) \mathrm{aC}$ & $0,00(0,00) \mathrm{aF}$ & $0,00(0,00) \mathrm{aD}$ \\
\hline Saflufenacil & 25 & $0,00(0,00)$ aA & $0,00(0,00) \mathrm{aB}$ & $0,00(0,00) \mathrm{aC}$ & $0,50(3,55) \mathrm{aBC}$ & $2,25(16,61) \mathrm{aDEF}$ & $2,75(16,48) \mathrm{aCD}$ \\
\hline Saflufenacil & 50 & $0,00(0,00) \mathrm{aA}$ & $0,00(0,00) \mathrm{aB}$ & $0,00(0,00) \mathrm{aC}$ & $0,50(3,55) \mathrm{bBC}$ & $2,75(17,81) \mathrm{aDEF}$ & $4,50(20,30) \mathrm{aC}$ \\
\hline Saflufenacil & 75 & $0,00(0,00) \mathrm{aA}$ & $0,00(0,00) \mathrm{aB}$ & $0,00(0,00) \mathrm{aC}$ & $4,50(21,21) \mathrm{aAB}$ & $3,50(14,20) \mathrm{aEF}$ & $5,75(23,75)$ aBC \\
\hline Saflufenacil & 100 & $0,00(0,00) \mathrm{aA}$ & $0,00(0,00) \mathrm{aB}$ & $0,00(0,00) \mathrm{aC}$ & $3,25(17,10)$ bABC & $4,00(19,98) \mathrm{abCDE}$ & $9,50(31,22) \mathrm{aBC}$ \\
\hline Saflufenacil & 125 & $0,00(0,00) \mathrm{aA}$ & $0,00(0,00) \mathrm{aB}$ & $0,00(0,00) \mathrm{aC}$ & $5,25(22,24) \mathrm{bAB}$ & $16,00(37,41) \mathrm{aC}$ & $16,75(42,10) \mathrm{aB}$ \\
\hline Saflufenacil & 150 & $0,00(0,00) \mathrm{aA}$ & $0,00(0,00) \mathrm{aB}$ & $0,00(0,00) \mathrm{aC}$ & $5,75(23,52) \mathrm{aA}$ & $9,25(30,49) \mathrm{aCDE}$ & $8,50(29,55) \mathrm{aBC}$ \\
\hline Saflufenacil & 175 & $0,00(0,00) \mathrm{aA}$ & $0,00(0,00) \mathrm{aB}$ & $0,00(0,00) \mathrm{aC}$ & $8,00(28,68) \mathrm{aA}$ & $9,00(29,97) \mathrm{aCDE}$ & $10,50(32,90) \mathrm{aBC}$ \\
\hline Saflufenacil & 200 & $0,00(0,00) \mathrm{aA}$ & $0,00(0,00) \mathrm{aB}$ & $0,00(0,00) \mathrm{aC}$ & $4,75(21,81) \mathrm{bAB}$ & $12,25(35,67) \mathrm{aCD}$ & $15,75(40,66) \mathrm{aB}$ \\
\hline Saflufenacil + Dash & 25 & $0,00(0,00) \mathrm{cA}$ & $31,25(59,30) \mathrm{bA}$ & $33,75(61,97) \mathrm{aB}$ & $1,25(11,07) \mathrm{bABC}$ & $44,00(70,12) \mathrm{aB}$ & $55,00(83,56) \mathrm{aA}$ \\
\hline Saflufenacil + Dash & 50 & $0,00(0,00) \mathrm{cA}$ & $31,35(59,30) \mathrm{bA}$ & $38,25(66,61) \mathrm{aA}$ & $2,25(15,00) \mathrm{bABC}$ & $61,25(89,90) \mathrm{aA}$ & $56,25(84,83)$ aA \\
\hline \multicolumn{2}{|l|}{ F tratamento $(\mathrm{T})$} & \multicolumn{3}{|c|}{$1.928,619^{* *}$} & \multicolumn{3}{|c|}{$59,927 * *$} \\
\hline \multicolumn{2}{|c|}{ F local de aplicação (L) } & \multicolumn{3}{|c|}{$1.081,816^{* *}$} & \multicolumn{3}{|c|}{$86,682 * *$} \\
\hline \multicolumn{2}{|l|}{$F(T) \times(L)$} & \multicolumn{3}{|c|}{$487,649 * *$} & \multicolumn{3}{|c|}{$13,230 * *$} \\
\hline \multicolumn{2}{|l|}{ d.m.s. (T) } & \multicolumn{3}{|c|}{2,21} & \multicolumn{3}{|c|}{13,80} \\
\hline \multicolumn{2}{|l|}{ d.m.s. (L) } & \multicolumn{3}{|c|}{3,06} & \multicolumn{3}{|c|}{19,11} \\
\hline \multicolumn{2}{|l|}{$\mathrm{CV}(\%)$} & \multicolumn{3}{|c|}{17,5} & \multicolumn{3}{|c|}{28,93} \\
\hline
\end{tabular}

Médias seguidas de mesma letra, maiúscula na linha e minúscula na coluna, não diferem estatisticamente entre si pelo teste de Tukey $(\mathrm{p}<0,05)$. ( ) Valor transformado pela equação " $\mathrm{y}=$ asen (raiz $(\mathrm{x} / 100))$ ". * Significativo a $5 \%$ de probabilidade. ${ }^{* *}$ Significativo a $1 \%$ de probabilidade. DAA: dias após aplicação. 
grandes injúrias às plantas. Ressalta-se que a adição do adjuvante Dash ao herbicida foi a responsável pela elevada fitotoxicidade, mesmo este aplicado em pequenas dosagens.

Notou-se aos 14 DAA (Tabela 3) que não houve diferenças nos sintomas de injúrias entre os tratamentos quanto à intoxicação das plantas; na associação de saflufenacil com Dash, na aplicação dirigida ao solo, a intoxicação nas plantas atingiu taxas menores que 4\%. Nas aplicações do herbicida isolado nas folhas houve diminuição quase total na intoxicação das plantas, com exceção do tratamento com a dose de $200 \mathrm{~g}$, no qual, ainda, verificaram-se $6,25 \%$ de fitotoxicidade. Já nas aplicações do herbicida em mistura com Dash, dirigidas à planta e ao solo + planta, a redução da fitoxicidade foi mais discreta, atingindo 41,25 e $44 \%$ com a dose de 25 g e 48,5 e $31,25 \%$ com a dose de $50 \mathrm{~g}$ do herbicida em mistura com Dash. Esses valores representam redução, em média, de 23,9 e $32,1 \%$, respectivamente, quando comparadas às porcentagens verificadas aos 7 DAA, atingindo até $97,4 \%$ quando a aplicação foi feita no solo. Esse resultado demonstra recuperação satisfatória das plantas quando o herbicida foi aplicado somente no solo, fato esse que pode ser atribuído a duas hipóteses: uma rápida absorção do herbicida pelas raízes, acompanhada de uma possivel lixiviação em curto prazo, não estando mais disponivel em quantidades prejudiciais no solo.

Os resultados observados aos 21 DAA estão apresentados na Tabela 3. Verificaramse leves sintomas de intoxicação (em média, $3 \%$ ) nas plantas com aplicação do herbicida isolado, nas doses de 25 a 100 g no solo - resultado também semelhante ao da aplicação do herbicida com a adição de Dash. Já nas maiores doses de saflufenacil utilizadas também em aplicação no solo (125, 150, 175 e 200 g) não se observaram diferenças entre as fitotoxicidades causadas por elas, sendo em média de $10 \%$. Com a aplicação dos tratamentos sobre a planta e solo + planta, não se observaram diferenças quando sem a adição de Dash. Verifica-se recuperação das plantas quando se compara com a avaliação realizada aos 7 DAA nos tratamentos com 25 e 50 g de saflufenacil com Dash, sendo em média de 29 e $26,5 \%$ a fitointoxicação nas aplicações nas plantas e em planta + solo, respectivamente, havendo superbrotação e bifurcação dos ponteiros das plantas.

Tabela 3 - Porcentagem de intoxicação de Eucalyptus urograndis submetido à aplicação de diferentes doses do herbicida saflufenacil, em três locais de aplicação na planta. Botucatu-SP, 2008

\begin{tabular}{|c|c|c|c|c|c|c|c|}
\hline \multirow{3}{*}{ Tratamento } & \multirow{2}{*}{ Dose } & \multicolumn{3}{|c|}{14 DAA } & \multicolumn{3}{|c|}{$21 \mathrm{DAA}$} \\
\hline & & \multicolumn{3}{|c|}{ Local de aplicação } & \multicolumn{3}{|c|}{ Local de aplicação } \\
\hline & $\left(\right.$ g i.a. ha $\left.^{-1}\right)$ & Solo & Planta & Solo + planta & Solo & Planta & Solo + planta \\
\hline Testemunha & - & $0,00(0,00) \mathrm{aE}$ & $0,00(0,00) \mathrm{aD}$ & $0,00(0,00) \mathrm{aD}$ & $0,00(0,00) \mathrm{aE}$ & $0,00(0,00) \mathrm{aD}$ & $0,00(0,00) \mathrm{aE}$ \\
\hline Saflufenacil & 25 & $0,75(6,05) \mathrm{aDE}$ & $1,00(8,56) \mathrm{aCD}$ & $1,75(12,55) \mathrm{aCD}$ & $1,50(10,41) \mathrm{bDE}$ & $4,50(21,01) \mathrm{aBC}$ & $4,25(20,23) a D$ \\
\hline Saflufenacil & 50 & $9,00(30,27)$ aA & $1,75(12,91)$ bBCD & $2,75(20,91)$ abBC & $2,75(16,04) \mathrm{bCD}$ & $1,25(11,06)$ bCD & $10,25(32,43) \mathrm{aBCD}$ \\
\hline Saflufenacil & 75 & $5,50(22,58)$ aABC & $1,50(11,87) \mathrm{bBCD}$ & $4,50(16,61) \mathrm{abC}$ & $3,25(17,89) \mathrm{aBCD}$ & $2,75(16,28) \mathrm{aBC}$ & $4,75(21,82) \mathrm{aCD}$ \\
\hline Saflufenacil & 100 & $5,00(21,99)$ abABC & $3,00(16,06) \mathrm{bBC}$ & $6,75(26,02) \mathrm{aBC}$ & $3,75(18,92) \mathrm{bBCD}$ & $4,50(21,01) \mathrm{bBC}$ & $10,25(31,39) \mathrm{aBCD}$ \\
\hline Saflufenacil & 125 & $1,00(10,02)$ bCDE & $1,00(10,02) b C D$ & $7,00(25,65) \mathrm{aBC}$ & $13,75(37,22)$ aA & $1,75(11,21) b C D$ & $8,75(29,70) \mathrm{aBCD}$ \\
\hline Saflufenacil & 150 & $5,75(23,86)$ aAB & $3,50(17,88) \mathrm{aBC}$ & $6,25(25,09) \mathrm{aBC}$ & $8,50(29,26) \mathrm{abAB}$ & $4,50(21,01) \mathrm{bBC}$ & $11,75(34,88) \mathrm{aBC}$ \\
\hline Saflufenacil & 175 & $7,25(27,07) \mathrm{aAB}$ & $3,25(17,97) \mathrm{aBC}$ & $6,00(24,71) \mathrm{aBC}$ & $7,25(27,12)$ aABC & $6,75(26,06) \mathrm{aB}$ & $11,50(34,57) \mathrm{aBC}$ \\
\hline Saflufenacil & 200 & $2,50(15,80)$ bBCD & $6,25(25,06)$ abB & $9,75(31,12) \mathrm{aB}$ & $6,75(26,02) \mathrm{bABC}$ & $8,50(28,48)$ abB & $13,00(36,77) \mathrm{aB}$ \\
\hline Saflufenacil + Dash & 25 & $1,25(7,90) \mathrm{cDE}$ & $41,25(77,03)$ aA & $34,00(62,13) \mathrm{bA}$ & $1,50(10,41) \mathrm{bDE}$ & $28,75(56,41)$ aA & $27,00(54,47)$ aA \\
\hline Saflufenacil + Dash & 50 & $3,75(19,18)$ cABCD & $48,50(69,66) \mathrm{aA}$ & $31,25(59,30) \mathrm{bA}$ & $4,50(20,78) \mathrm{bBCD}$ & $30,50(58,44)$ aA & $26,25(53,65) \mathrm{aA}$ \\
\hline \multicolumn{2}{|l|}{$\mathrm{F}$ tratamento $(\mathrm{T})$} & \multicolumn{3}{|c|}{$76,008^{* *}$} & \multicolumn{3}{|c|}{$53,382 * *$} \\
\hline \multicolumn{2}{|c|}{ F local de aplicação (L) } & \multicolumn{3}{|c|}{$39,084^{* *}$} & \multicolumn{3}{|c|}{$52,870^{* *}$} \\
\hline \multicolumn{2}{|l|}{$\mathrm{F}(\mathrm{T}) \mathrm{x}(\mathrm{L})$} & \multicolumn{3}{|c|}{$23,196^{* *}$} & \multicolumn{3}{|c|}{$14,577 * *$} \\
\hline \multicolumn{2}{|l|}{ d.m.s. (T) } & \multicolumn{3}{|c|}{9,92} & \multicolumn{3}{|c|}{9,52} \\
\hline \multicolumn{2}{|l|}{ d.m.s. (L) } & \multicolumn{3}{|c|}{13,73} & \multicolumn{3}{|c|}{13,19} \\
\hline \multicolumn{2}{|l|}{$\mathrm{CV}(\%)$} & \multicolumn{3}{|c|}{25,7} & \multicolumn{3}{|c|}{22,4} \\
\hline
\end{tabular}

Médias seguidas de mesma letra, maiúscula na linha e minúscula na coluna, não diferem estatisticamente entre si pelo teste de Tukey $(\mathrm{p}<0,05)$. ( ) Valor transformado pela equação " $\mathrm{y}=$ asen (raiz $(\mathrm{x} / 100))$ ". * Significativo a $5 \%$ de probabilidade. ** Significativo a $1 \%$ de probabilidade. DAA: dias após aplicação. 
Aos 42 DAA (Tabela 4), as plantas não apresentaram sintomas de intoxicação nos tratamentos com o herbicida isolado, independentemente da dose utilizada, havendo recuperação total delas. Entretanto, nos tratamentos com o herbicida associado com Dash, as injúrias mantiveram-se, porém em menor escala, nas aplicações realizadas na planta: 3,75 e $8 \%$, com as doses de 25 e 50 g, respectivamente. Nas aplicações realizadas em planta + solo, não se observaram diferenças na fitotoxicidade proporcionada pelas doses de saflufenacil (25 e $50 \mathrm{~g}$ ) com a adição de Dash, sendo em média de $8,5 \%$. Aos 56 DAA (Tabela 4), não foi observada fitointoxicação em nenhum dos tratamentos, havendo, assim, recuperação visual total das plantas.

Corroborando esses resultados, Ribeiro \& Oliveira (1987), em aplicação de oxyfluorfen no solo em plantios de eucalipto, avaliaram o seu efeito sobre o desenvolvimento das mudas. Esses pesquisadores verificaram que os resultados variam de acordo com a espécie; Eucalyptus camaldulensis e E. tereticornis foram altamente tolerantes e não apresentaram fitointoxicação. Já as espécies E. citriodora, E. resinifolia e E. intermedia apresentaram queima dos tecidos apicais, mas recuperaram-se com facilidade. E. pellita e E. urophyla sofreram danos ligeiramente mais severos, e E. pilularis e E. cloeziana, danos mais graves, com algumas plantas chegando a morrer.

Na Tabela 5 estão apresentados os dados de altura de plantas e massa seca da parte aérea, os quais diferiram entre as doses de saflufenacil aplicadas isoladas e com a adição do adjuvante Dash, bem como para a variável local de aplicação na planta e na interação doses x local de aplicação. Não foram verificadas diferenças no diâmetro, mostrando resultados semelhantes aos da testemunha.

Não foram observadas diferenças na altura de plantas com a aplicação das doses do herbicida isolado, independentemente do local de aplicação. Entretanto, nas aplicações do herbicida nas doses de 25 e 50 g com a adição de Dash sobre planta + solo, verificou-se redução de 9,75 e 11,5\% na altura, respectivamente, em relação às plantas testemunhas. Comparando esses mesmos tratamentos, mas com aplicação somente no solo, observou-se diminuição da altura de 7,9 e 7,6\% (doses de 25 e 50 g, com adição de Dash, respectivamente). Com a aplicação somente na planta dos tratamentos com saflufenacil (doses de 25e 50 g, com adição de Dash), notou-se redução de 10,2 e 9\% na altura das plantas, em relação àquelas com aplicação em planta + solo.

Concordando os resultados de altura, não se observaram diferenças entre as massas secas da parte aérea que receberam aplicação no solo ou na planta do herbicida isolado, independentemente das doses utilizadas, com exceção da dose de 200 g, que reduziu em 19\% a massa seca das plantas, quando aplicada sobre planta + solo.

Nas aplicações sobre planta + solo com 25 e $50 \mathrm{~g}$ de saflufenacil em associação com Dash, observaram-se reduções na massa seca, em média, de 33 e $38 \%$, respectivamente. Também foram verificadas diferenças em média de $26 \%$ com a aplicação desses mesmos tratamentos no solo ou na planta em relação às aplicações realizadas no solo + planta. Tuffi Santos et al. (2006b) observaram redução de massa seca em diferentes espécies de eucalipto, porém com o herbicida glyphosate.

Esses resultados podem ser justificados pelas injúrias severas observadas nas primeiras semanas de avaliação, provocadas após a aplicação de saflufenacil + Dash, o que possivelmente resultou em redução de crescimento e desenvolvimento das plantas em decorrência da queima de ponteiros e necrose das folhas, principalmente as mais velhas, as quais não se recuperaram, o que teve consequência direta sobre a massa seca.

Aos 56 dias após aplicação, como os sintomas visuais de fitotointoxicação não foram mais observados, o crescimento das plantas pode não ter sido comprometido, pois houve superbrotação e bifurcação dos ponteiros, fato esse que prejudica a qualidade final da madeira, mas contribui para a recuperação da altura e massa verde das plantas. As taxas de absorção e translocação foliar dos herbicidas e, consequentemente, sua eficácia estão diretamente ligadas aos tipos de estrutura encontrados na folha e à permeabilidade das cutículas, as quais dependem de sua constituição e polaridade (Baker, 1982), o que 
Tabela 4 - Porcentagem de fitotoxicidade nas plantas de Eucalyptus urograndis submetidas à aplicação de diferentes doses do herbicida saflufenacil, em três locais de aplicação. Botucatu-SP, 2008

\begin{tabular}{|c|c|c|c|c|c|c|c|}
\hline \multirow{3}{*}{ Tratamento } & \multirow{2}{*}{ Dose } & \multicolumn{3}{|c|}{$42 \mathrm{DAA}$} & \multicolumn{3}{|c|}{$56 \mathrm{DAA}$} \\
\hline & & \multicolumn{3}{|c|}{ Local de aplicação } & \multicolumn{3}{|c|}{ Local de aplicação } \\
\hline & $\left(\right.$ g i.a. ha $\left.^{-1}\right)$ & Solo & Planta & Solo + Planta & Solo & Planta & Solo + Planta \\
\hline Testemunha & - & $0,00(0,00) \quad \mathrm{aA}$ & $0,00(0,00) \quad \mathrm{aC}$ & $0,00(0,00) \quad \mathrm{aB}$ & 0,00 & 0,00 & 0,00 \\
\hline Saflufenacil & 25 & $0,00(0,00) \quad \mathrm{aA}$ & $0,00(0,00) \quad \mathrm{aC}$ & $0,00(0,00) \quad \mathrm{aB}$ & 0,00 & 0,00 & 0,00 \\
\hline Saflufenacil & 50 & $0,00(0,00) \quad \mathrm{aA}$ & $0,00(0,00) \quad \mathrm{aC}$ & $0,00(0,00) \quad \mathrm{aB}$ & 0,00 & 0,00 & 0,00 \\
\hline Saflufenacil & 75 & $0,00(0,00) \quad \mathrm{aA}$ & $0,00(0,00) \quad \mathrm{aC}$ & $0,00(0,00) \quad \mathrm{aB}$ & 0,00 & 0,00 & 0,00 \\
\hline Saflufenacil & 100 & $0,00(0,00) \quad \mathrm{aA}$ & $0,00(0,00) \quad \mathrm{aC}$ & $0,00(0,00) \quad \mathrm{aB}$ & 0,00 & 0,00 & 0,00 \\
\hline Saflufenacil & 125 & $0,00(0,00) \quad \mathrm{aA}$ & $0,00(0,00) \quad \mathrm{aC}$ & $0,00(0,00) \quad \mathrm{aB}$ & 0,00 & 0,00 & 0,00 \\
\hline Saflufenacil & 150 & $0,00(0,00) \quad \mathrm{aA}$ & $0,00(0,00) \quad \mathrm{aC}$ & $0,00(0,00) \quad \mathrm{aB}$ & 0,00 & 0,00 & 0,00 \\
\hline Saflufenacil & 175 & $0,00(0,00) \quad \mathrm{aA}$ & $0,00(0,00) \quad \mathrm{aC}$ & $0,00(0,00) \quad \mathrm{aB}$ & 0,00 & 0,00 & 0,00 \\
\hline Saflufenacil & 200 & $0,00(0,00) \quad \mathrm{aA}$ & $0,00(0,00) \quad \mathrm{aC}$ & $0,00(0,00) \quad \mathrm{aB}$ & 0,00 & 0,00 & 0,00 \\
\hline Saflufenacil + Dash & 25 & $0,00(0,00) \quad \mathrm{cA}$ & $3,75(16,91) \quad b B$ & $8,00(28,68) \quad \mathrm{aA}$ & 0,00 & 0,00 & 0,00 \\
\hline Saflufenacil + Dash & 50 & $0,00(0,00) \quad \mathrm{bA}$ & $8,00(28,68) \quad \mathrm{aA}$ & $9,00(30,43) \quad \mathrm{aA}$ & 0,00 & 0,00 & 0,00 \\
\hline $\mathrm{F}$ tratamento $(\mathrm{T})$ & \multicolumn{4}{|c|}{$153,455^{* *}$} & \multicolumn{3}{|c|}{-} \\
\hline F local de aplicação (L) & \multicolumn{4}{|c|}{$87,729 * *$} & \multicolumn{3}{|c|}{-} \\
\hline$F(T) \times(L)$ & \multicolumn{4}{|c|}{$41,506^{* *}$} & \multicolumn{3}{|c|}{$=$} \\
\hline d.m.s. (T) & \multicolumn{4}{|c|}{3,36} & \multicolumn{3}{|c|}{ - } \\
\hline d.m.s. (L) & \multicolumn{4}{|c|}{4,67} & \multicolumn{3}{|c|}{-} \\
\hline $\mathrm{CV}(\%)$ & \multicolumn{4}{|c|}{62,8} & \multicolumn{3}{|c|}{-} \\
\hline
\end{tabular}

Médias seguidas de mesma letra, maiúscula na linha e minúscula na coluna, não diferem estatisticamente entre si pelo teste de Tukey $(\mathrm{p}<0,05)$. ( ) Valor transformado pela equação " $\mathrm{y}=$ asen (raiz $(\mathrm{x} / 100))$ ". * Significativo a $5 \%$ de probabilidade. ** Significativo a $1 \%$ de probabilidade. DAA: dias após aplicação.

Tabela 5 - Médias de altura (cm), diâmetro de caule ( $\mathrm{mm})$ e massa seca das plantas $(\mathrm{g})$ de Eucalyptus urograndis submetidas à aplicação de diferentes doses do herbicida saflufenacil, em três locais de aplicação. Botucatu-SP, 2008

\begin{tabular}{|c|c|c|c|c|c|c|c|c|c|c|}
\hline \multirow{3}{*}{ Tratamento } & \multirow{3}{*}{$\begin{array}{c}\text { Dose } \\
\left(\mathrm{g} \text { i.a. } \mathrm{ha}^{-1}\right)\end{array}$} & \multicolumn{3}{|c|}{ Altura $(\mathrm{cm})$} & \multicolumn{3}{|c|}{ Diâmetro de caule $(\mathrm{mm})$} & \multicolumn{3}{|c|}{ Massa Seca $(g)$} \\
\hline & & \multicolumn{3}{|c|}{ Local de aplicação } & \multicolumn{3}{|c|}{ Local de aplicação } & \multicolumn{3}{|c|}{ Local de aplicação } \\
\hline & & Solo & Planta & Solo + Planta & Solo & Planta & $\begin{array}{l}\text { Solo }+ \\
\text { Planta }\end{array}$ & Solo & Planta & $\begin{array}{l}\text { Solo }+ \\
\text { Planta }\end{array}$ \\
\hline Testemunha & - & $51,50 \mathrm{aA}$ & $51,50 \mathrm{aA}$ & $51,50 \mathrm{abA}$ & 2,96 & 2,96 & 2,96 & $13,22 \mathrm{aA}$ & $13,22 \mathrm{aA}$ & $13,22 \mathrm{aA}$ \\
\hline Saflufenacil & 25 & $50,50 \mathrm{aA}$ & $51,25 \mathrm{aA}$ & $54,25 \mathrm{aA}$ & 2,66 & 2,79 & 2,70 & $12,55 \mathrm{aA}$ & $12,64 \mathrm{abA}$ & $13,20 \mathrm{aA}$ \\
\hline Saflufenacil & 50 & $51,00 \mathrm{aA}$ & 50,25 aA & $52,25 \mathrm{aA}$ & 2,95 & 2,57 & 3,12 & $12,42 \mathrm{aA}$ & $12,50 \mathrm{abA}$ & $12,81 \mathrm{abA}$ \\
\hline Saflufenacil & 75 & $50,25 \mathrm{aA}$ & 50,25 aA & 49,50 abcA & 2,84 & 2,63 & 2,58 & $11,89 \mathrm{aA}$ & $12,20 \mathrm{abA}$ & 12,19 abA \\
\hline Saflufenacil & 100 & $52,25 \mathrm{aA}$ & $51,50 \mathrm{aA}$ & 50,50 abcA & 2,97 & 2,87 & 2,81 & 11,75 aA & $12,17 \mathrm{abA}$ & $12,16 \mathrm{abA}$ \\
\hline Saflufenacil & 125 & $50,00 \mathrm{aA}$ & $52,00 \mathrm{aA}$ & 53,50 abcA & 3,03 & 2,83 & 2,79 & $12,23 \mathrm{aA}$ & $12,44 \mathrm{abA}$ & 11,14 abcA \\
\hline Saflufenacil & 150 & $49,25 \mathrm{aA}$ & $51,75 \mathrm{aA}$ & $52,25 \mathrm{aA}$ & 2,57 & 2,67 & 2,93 & $11,47 \mathrm{aA}$ & $12,60 \mathrm{abA}$ & 11,49 abcA \\
\hline Saflufenacil & 175 & $50,00 \mathrm{aB}$ & $54,50 \mathrm{aA}$ & $51,50 \mathrm{abAB}$ & 2,59 & 3,02 & 2,73 & $12,26 \mathrm{aA}$ & $11,83 \mathrm{abA}$ & 11,27 abcA \\
\hline Saflufenacil & 200 & $49,75 \mathrm{aB}$ & 55,25 aA & $50,75 \mathrm{abcB}$ & 2,57 & 2,82 & 3,07 & 11,70 aA & $11,70 \mathrm{abA}$ & $10,71 \mathrm{bcdA}$ \\
\hline Saflufenacil + Dash & 25 & $50,50 \mathrm{aA}$ & $51,75 \mathrm{aA}$ & $46,50 \mathrm{bcB}$ & 2,76 & 2,70 & 2,44 & $12,87 \mathrm{aA}$ & $10,96 \mathrm{abA}$ & $8,86 \mathrm{cdB}$ \\
\hline Saflufenacil + Dash & 50 & $49,25 \mathrm{aAB}$ & $50,00 \mathrm{aA}$ & $45,50 \mathrm{cB}$ & 2,80 & 3,13 & 2,62 & $11,99 \mathrm{aA}$ & $10,35 \mathrm{bA}$ & $8,20 \mathrm{~dB}$ \\
\hline F Tratamento (T) & \multicolumn{4}{|c|}{$3,098^{* *}$} & \multicolumn{3}{|c|}{$1,053^{\text {ns }}$} & \multicolumn{3}{|c|}{$6,917^{* *}$} \\
\hline F Local de aplicação (L) & \multicolumn{4}{|c|}{$4,365 * *$} & \multicolumn{3}{|c|}{$0,103^{\mathrm{ns}}$} & \multicolumn{3}{|c|}{$6,326^{* *}$} \\
\hline $\mathrm{F}(\mathrm{T}) \times(\mathrm{L})$ & \multicolumn{4}{|c|}{$2,312 * *$} & \multicolumn{3}{|c|}{$1,625^{\mathrm{ns}}$} & \multicolumn{3}{|c|}{$2,299 * *$} \\
\hline d.m.s. (T) & \multicolumn{4}{|c|}{5,53} & \multicolumn{3}{|c|}{0,07} & \multicolumn{3}{|c|}{2,64} \\
\hline d.m.s. (L) & \multicolumn{4}{|c|}{3,99} & \multicolumn{3}{|c|}{0,05} & \multicolumn{3}{|c|}{1,91} \\
\hline $\mathrm{CV}(\%)$ & \multicolumn{4}{|c|}{4,7} & \multicolumn{3}{|c|}{11,2} & \multicolumn{3}{|c|}{9,6} \\
\hline
\end{tabular}

Médias seguidas de mesma letra, maiúscula na linha e minúscula na coluna, não diferem estatisticamente entre si pelo teste de Tukey $(\mathrm{p}<0,05)$. ( ) Valor transformado pela equação " $\mathrm{y}=$ asen (raiz $(\mathrm{x} / 100))$ ". * Significativo a $5 \%$ de probabilidade. ** Significativo a $1 \%$ de probabilidade. DAA: dias após aplicação. 
poderia explicar esse restabelecimento das plantas, ou seja, o desaparecimento total de injúrias provocadas pelo herbicida saflufenacil, aplicado em diferentes doses, com ou sem adição do adjuvante Dash.

Pode-se inferir que o herbicida saflufenacil, isolado ou associado com Dash, não causou prejuízos à cultura de E. urograndis quando aplicado no solo independentemente da dose utilizada, bem como a sua aplicação na parte aérea sem associação com Dash.

\section{LITERATURA CITADA}

BAKER, E. A. Chemistry and morphology of plant epicuticular waxes. In: CUTLER, D. F.; ALVIN, K. L.; PRICE, C. E. (Eds.). The plant cuticle. London: Academic Press, 1982. p. 140-161.

BASF. Disponível em: <http://agproducts.basf.us/products/ kixor-herbicide.html>. Acesso em: 7 dez. 2010.

CAMARGO, P. N. Seletividade. B. Inf. IPEF, v. 5, n. 15, p. 168-94, 1977.

CANTARELLI, E. B. et al. Efeito do manejo de plantas daninhas no desenvolvimento inicial de Pinus taeda em várzeas na argentina. R. Árvore, v. 30, n. 5, p. 711-718, 2006.

CONSTANTIN, J.; OLIVEIRA JR., R. S.; MACIEL, C. D. G. Azafenidin: novo herbicida para o controle de plantas daninhas em Eucalyptus camaldulensis. Planta Daninha, v. 18 , n. 3, p. $405-411,2000$

DAVIS, V. M. et al. Fall and spring preplant herbicide applications influence spring emergence of glyphosateresistant horseweed (Conyza canadensis). Weed Technol., v. 24, n. 1, p. 11-10, 2010.

GARCIA, J. N.; SOUZA, M. P; ANTUNES Jr, R. O. Combate a matocompetição em povoamentos de pinus. Informativo ARESB. Disponível em: $<\mathrm{http}$ // www.aresb.com.br/informativoaresb/jornal/boletins/2007/pdf/ informativo_maio.pdf+aresb+pinu>. Acesso em: 10 ago. 2010.
GEIER, P. W.; STAHLMAN, P. W.; CHARVAT, L. D. Dose responses of five broadleaf weeds to saflufenacil. Weed Technol., v. 23, n. 2, p. 313-316, 2009.

GROSSMANN, K. et al. The herbicide saflufenacil (KixorTM) is a new inhibitor of protoporphyrinogen IX oxidase activity. Weed Sci., v. 58, n. 1, p. 1-9, 2010 .

RIBEIRO, G. T.; OLIVEIRA, A. C. Uso de herbicidas em reflorestamentos no cerrado. Série Técnica IPEF, v. 4, n. 12, p. 116-131, 1987.

SOCIEDADE BRASILEIRA DA CIÊNCIA DAS PLANTAS DANINHAS - SBCPD. Procedimentos para instalação, avaliação e análise de experimentos com herbicidas. Londrina: 1995. $42 \mathrm{p}$.

SOLTANI, N.; SHROPSHIRE, C.; SIKKEMA, P. H. Sensitivity of leguminous crops to saflufenacil. Weed Technol., v. 24, n. 2, p. 143-146, 2010.

SOLTANI, N.; SHROPSHIRE, C.; SIKKEMA, P. H. Response of corn to pre emergence and postemergence applications of saflufenacil. Weed Technol., v. 23, n. 3, p. 331-334, 2009.

TOLEDO, R. E. B. et al. Faixas de controle de plantas daninhas e seus reflexos no crescimento de plantas de eucalipto. Sci. For., v. 26, n. 64, p. 78-92, 2003.

TUFFI SANTOS, L. D. et al. Danos visuais e anatômicos causados pelo glyphosate em folhas de Eucalyptus grandis. Planta Daninha, v. 26, n. 1, p. 9-16, 2008.

TUFFI SANTOS, L. D. et al. Intoxicação de espécies de eucalipto submetidas a deriva do glyphosate.

Planta Daninha, v. 24, n. 2, p. 359-364, 2006 a.

TUFFI SANTOS, L. D. et al. Intoxicação de eucalipto submetido à deriva simulada de diferentes herbicidas.

Planta Daninha, v. 24, n. 3, p. 521-526, 2006 b.

VICTORIA FILHO, R. Tipos de herbicidas para uso em florestas. Série Técnica IPEF, v. 4, n. 12, p. 36-44, 1987. 